\title{
Emerging concepts in synucleinopathies
}

\author{
Tiago Fleming Outeiro ${ }^{1,2,3,4,5}$
}

Received: 12 February 2021 / Revised: 12 February 2021 / Accepted: 12 February 2021 / Published online: 24 February 2021

(c) The Author(s), under exclusive licence to Springer-Verlag GmbH, DE part of Springer Nature 2021

\begin{abstract}
Alpha-synuclein (aSyn) is a central player in age-associated disorders known as synucleinopathies, where it accumulates in intracellular protein inclusions. Synucleinopathies include Parkinson's disease (PD), Dementia with Lewy bodies (DLB), and multiple systems atrophy (MSA), all of which are highly debilitating age-associated disorders. Neuropathology studies show us that the old brain is multi-morbid, with the simultaneous accumulation of various types of protein pathologies that have been, traditionally, associated with individual diseases [1]. We assume that during the process of protein aggregation, different types of aggregated species (oligomers, protofibrils, fibrils, etc) are formed, and that at least some might be toxic to cells/tissues/organs, thereby leading to neurodegeneration. However, we still cannot definitively establish this causal relationship between protein aggregation and disease.
\end{abstract}

Strikingly, our understanding of the normal biology/function of the proteins which aggregate in neurodegeneration is still elusive, and has not received as much attention as that devoted to the study of the process of aggregation itself. This is particularly relevant in synucleinopathies, as we lack detailed understanding of the function and "life-cycle"

Tiago Fleming Outeiro

touteir@gwdg.de

1 Department of Experimental Neurodegeneration, Center for Biostructural Imaging of Neurodegeneration, University Medical Center, Göttingen, Germany

2 Cluster of Excellence "Multiscale Bioimaging: from Molecular Machines to Networks of Excitable Cells" (MBExC), University of Göttingen, Göttingen, Germany

3 Max Planck Institute for Experimental Medicine, Göttingen, Germany

4 Faculty of Medical Sciences, Translational and Clinical Research Institute, Newcastle University, Framlington Place, Newcastle Upon Tyne NE2 4HH, UK

5 Scientific Employee With a Honorary Contract At Deutsches Zentrum Für Neurodegenerative Erkrankungen (DZNE), Göttingen, Germany of aSyn, and of the molecular underpinnings leading to neurodegeneration.

Since the synuclein "journey" will soon be turning 25 , it is important to introduce and discuss novel concepts, and to define where we are so can have a clear view of where we need to go [2].

This cluster comprises articles that review emerging aspects of synucleinopathies that deserve further attention in order to enable us to identify mechanisms and targets of interest for improved diagnostics and therapeutic interventions.

Brás and colleagues start off by assessing the pathogenicity and roles of genetic variants in the various synuclein genes ( $S N C A, S N C B$, and $S N C G$ ) associated with synucleinopathies [3]. Although the three synucleins share a high homology degree, and seem to be enriched at presynaptic terminals, only aSyn has been unequivocally associated with synucleinopathies.

Genetic studies on the association of $S N C B$ with synucleinopathies have yielded inconclusive results. Interestingly, recent evidence suggests that, at least in laboratory models, beta-synuclein bSyn might also cause cellular dysfunction and neurodegeneration $[4,5]$. No studies have found an association between $S N C G$ mutations and synucleinopathies yet.

The function of aSyn has been related to different steps of intracellular trafficking. In fact, as a presynaptic protein, aSyn has been strongly implicated in the regulation of synaptic vesicle biology. However, the occurrence of aSyn in other tissues, including certain types of blood cells, raises questions about the actual physiological function of the protein. Based on recent studies on the composition and structure of Lewy bodies, and on a growing body of genetic and cell biology data, Fanning and colleagues disccuss the idea that synucleinopathies involve disruptions in both lipid and trafficking homeostasis [6].

Erskine and colleagues extend this idea by noticing that, upon neuropathological analyses, disorders that are not traditionally classified as synucleinopathies, appear to accumulate disproportionately high levels of LBs [7]. A closer look at such disorders, which include lysosomal storage 
disorders, iron storage disorders and mitochondrial diseases, suggests that the process of LB formation may result from perturbations in important biological pathways involved in those conditions, i.e., autophagic function, metal and lipid homeostasis, and mitochondrial function. Strikingly, all of these pathways have been implicated in PD, suggesting aSyn accumulation may arise due to alterations in basic biological processes.

PD and other synucleinopathies are now known to be systemic disorders, manifesting with features that reflect the involvement of peripheral systems, such as the gut and the immune system. This recent, and more holistic view of PD, is discussed by Harms and colleagues [8]. In particular, they argue that PD-associated immune responses impact on neuronal health, and that such responses occur also in the periphery, involving both innate as well as adaptive systems. Importantly, aSyn appears to play a central role in modulating these two types of immune responses, possible by triggering neuroinflammatory processes.

Is aSyn aggregation a culprit or a bystander in synucleinopathies? What molecular factors trigger and modulate aSyn aggregation? How do LBs and other types of aSyn pathology form? What is the role of posttranslational modifications in modulating the structure, biological function, subcellular distribution, and aggregation of aSyn? How does the immune system sense and react to different forms (aggregated, posttranslationally-modified, lipid/membrane-bound, etc.) of aSyn? Does aSyn pathology "spread" in the brain, or between the brain and other organs? These are only but a few open questions that have entertained, and continue to entertain armies of scientists throughout the world. Sadly, despite a tremendous amount of knowledge about aSyn, our progress towards the goal we all aim for, i.e., better therapeutic strategies for patients, has been slow. Patients would argue too slow. Therefore, we need more critical and more open views that should enable us to integrate novel ideas, novel concepts, and novel models, with the ultimate goal of developing better tools to define, diagnose, and treat synucleinopathies, a group of devastating conditions associated with a protein that may, or may not, be at fault.

We hope you enjoy this cluster of reviews by leading authors in the field, and that they inspire the community to tackle open and important questions in the field.
Acknowledgements TFO is supported by the Deutsche Forschungsgemeinschaft (DFG, German Research Foundation) under Germany's Excellence Strategy_EXC 2067/1-390729940).

Author contributions TFO wrote the manuscript.

Funding TFO is supported by the Deutsche Forschungsgemeinschaft (DFG, German Research Foundation) under Germany's Excellence Strategy-EXC 2067/1 390729940.

\section{Compliance with ethical standard}

Conflict of interest The authors declare that they have no conflict of interest.

\section{References}

1. Attems J (2017) The multi-morbid old brain. Acta Neuropathol 134:169-170

2. Brás IC, Dominguez-Meijide A, Gerhardt E et al (2020) Synucleinopathies: where we are and where we need to go. J Neurochem 153:433-454

3. Brás J, Gibbons E, Guerreiro R (2020) Genetics of synucleins in neurodegenerative diseases. Acta Neuropathol. https://doi. org/10.1007/s00401-020-02202-1

4. Tenreiro S, Rosado-Ramos R, Gerhardt E, Favretto F, Magalhães F, Popova B, Becker S, Zweckstetter M, Braus GH, Outeiro TF (2016) Yeast reveals similar molecular mechanisms underlying alpha- and beta-synuclein toxicity. Hum Mol Genet 25(2):275-290

5. Taschenberger G, Toloe J, Tereshchenko J, Akerboom J, Wales P, Benz R, Becker S, Outeiro TF, Looger LL, Bähr M, Zweckstetter M, Kügler S (2013) $\beta$-synuclein aggregates and induces neurodegeneration in dopaminergic neurons. Ann Neurol 74(1):109-118

6. Fanning S, Selkoe D, Dettmer U (2020) Vesicle trafficking and lipid metabolism in synucleinopathy. Acta Neuropathol. https:// doi.org/10.1007/s00401-020-02177-z.

7. Erskine D, Koss D, Korolchuk VI, Outeiro TF, Attems J, McKeith I (2021) Lipids, lysosomes and mitochondria: insights into Lewy body formation from rare monogenic disorders. Acta Neuropathol. https://doi.org/10.1007/s00401-021-02266-7

8. Harms AS, Ferreira SA, Romero-Ramos M (2021) Periphery and brain, innate and adaptive immunity in Parkinson's disease. Acta Neuropathol. https://doi.org/10.1007/s00401-021-02268-5

Publisher's Note Springer Nature remains neutral with regard to jurisdictional claims in published maps and institutional affiliations. 